\title{
Multivariate analyses of the Lifeways cross-generation study of BMI
}

\author{
G.E. Kelly ${ }^{1}$, Q. Zhang ${ }^{1}$, C. Murrin ${ }^{2}$ and C. Kelleher ${ }^{2}$ \\ ${ }^{1}$ School of Mathematics and Statistics and ${ }^{2}$ School of Public Health, Physiotherapy and Sports Science, University \\ College Dublin, Dublin 4, Ireland.
}

Increasing levels of body mass index (BMI) present a problem, particularly in children. Determining possible pathways of familial transmission is important, both for prevention and management intervention ${ }^{(1,2,3)}$. The aim of the present analysis was to demonstrate the use of multivariate statistical models to examine familial patterns in the Lifeways cross-generation cohort study.

Expectant mothers were recruited initially in $2001-2003^{(4)}$. In addition to their index infant, mothers recruited if possible their partner and at least one living grandparent. There were 1094 children born to 1082 mothers. Height and weight at age 5 were recorded for 570 children. Where available from either baseline or follow-up, BMI measurements of their parents, maternal and paternal grandparents were included. The data are multivariate in that there are up to seven variables corresponding to family members' BMI. We used principal component analysis (PCA) to reduce the dimensionality of the data ${ }^{(5)}$. We then used latent variable models in the form of factor analysis and structural equation models (SEM) to describe the structure of the covariance matrix of the seven variables ${ }^{(5)}$. All models were fitted using the lavaan package in $\mathrm{R}^{(6)}$. Missing data were imputed ${ }^{(7)}$.

With PCA, two components accounted for $68 \%$ of variation in the data. These were firstly overall level of BMI and then variation between individuals in the maternal family (including the child) and paternal family. With factor analysis, there were again two factors interpreted as maternal family and paternal family (including the child). SEM was then used to test a specific pathway- the intrauterine effect/ mtDNA effect on BMI. Maternal grandmother, mother, child comprised one factor and paternal grandmother, father comprised the second factor (Fig. 1). The loadings of the second factor were not significant but when the father was omitted from the second factor, the fit was better, providing confirmation of the model.

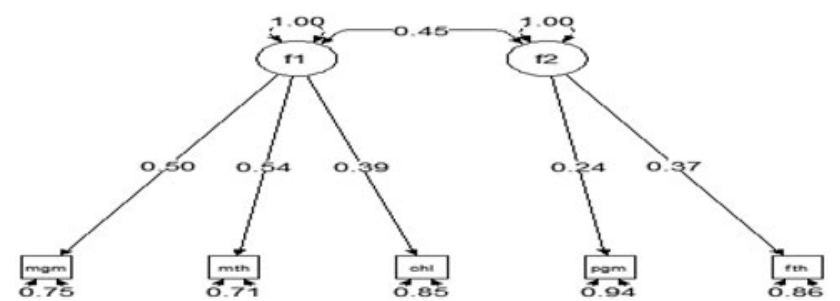

Fig. 1. Path diagram for intrauterine/mtDNA effect. $\mathrm{f} 1$ and $\mathrm{f} 2$ are latent factors. The numbers denote factor loadings. $\mathrm{mgm} / \mathrm{pgm}=\mathrm{maternal} / \mathrm{paternal}$ grandmother.

In conclusion, these analyses confirmed particularly maternal-line patterns associated with child's BMI, consistent with intrauterine exposure, as well as shared familial or environmental exposures.

1. Murrin CM, Kelly GE, Tremblay RE et al. (2012) BMC Public Health 12, 1-11.

2. Davey Smith G, Steer C, Leary S et al. (2007) Arch Dis Child 92, 876-80.

3. Kelly GE, Murrin C, Viljoen K et al. (2014) BMJ Open 4, e005732.

4. O'Mahony D, Fallon UB, Hannon F et al. (2007) Ir Med J 100, 3-6.

5. Everitt B \& Duun G (1991) Applied Multivariate Data Analysis. London: Edward Arnold.

6. Rosseel Y (2012) J Stat Softw 48, 1-36.

7. Josse J \& Husson F (2016) J Stat Softw 70, 1-31. 